\title{
B2. INHIBITION OF THE MYOTOXICITY INDUCED BY CROTOXIN B, FROM Crotalus durissus terrificus VENOM, BY CAMELID NANOBODIES.
}

Marcos Barros Luiz ${ }^{1}$; Soraya dos Santos Pereira ${ }^{1}$; Naan Rodrigues Gonçalves ${ }^{1}$; Nidiane Dantas Reis Prado${ }^{1}$; Leandro Soares Moreira Dill ${ }^{1}$; Andreimar Martins Soares ${ }^{1}$; Fernando Berton Zanchi ${ }^{1}$; Juliana Pavan Zuliani'; Rodrigo Guerino Stábeli²; Carla Freire Celedônio Fernandes ${ }^{3}$.

${ }^{1}$ Fiocruz - RO;

${ }^{2}$ Fiocruz;

${ }^{3}$ Fiocruz - RO, 2Centro de Pesquisa em Medicina Tropical - CEPEM.

INTRODUCTION In addition to conventional immunoglobulins $\mathrm{G}$, camelids produce functional antibodies devoid of light chains and $\mathrm{CH} 1$ domains, called heavy-chain camelid antibodies (HCAbs). The antigen recognition site of HCAbs is formed by the single domain referred to as $\mathrm{VHH}$ or nanobody (Nbs). Besides thermal and $\mathrm{pH}$ stability, important for field treatment, nanobodies have one tenth the size of conventional antibodies, possess low immunogenicity, can be produced in microorganisms and are able to neutralize animal toxins, allowing its use as a tool in the treatment of snakebite envenoming. The genus Crotalus accounts for about 7.5\% of snakebite accidents in Brazil and its mortality rate can reach 4.7\%. Neurotoxic, nephrotoxic and myotoxic effects of Crotalus envenoming are mainly related to the crotoxin (CTX), a heterodimer formed via noncovalent interactions between the phospholipase A2 (CB, crotoxin-B, PLA2-CB) basic and enzymatically active, and crotapotin ( $\mathrm{CA}$, crotoxin-A), acid component and enzymatically inactive. The treatment is performed by administering immunobiologics derived from hyperimmunized horses. Besides high cost of production, the serum therapy is less effective in neutralizing toxins in deep tissues and can cause adverse reactions. Thus, the search for complementary methods in cases of snakebite envenoming has been increasing.

OBJECTIVE Exploring the advantages of nanobody, this work aimed to characterize in silico, in vitro and in vivo clones previously selected against crotoxin, from a llama VHH library.

METHODOLOGY The molecular docking was used for demonstrate in silico interaction between $\mathrm{Nbs}$ and $\mathrm{CB}$ subunit and affinity constants were determined by 
surface plasmon resonance (SPR). In vitro inhibition of CB and CTX phospholipase A2 activity was performed using synthetic fluorescent phospholipid acyl-NBD-PE and in vivo neutralization of myotoxic effect induced by crotoxin-B in mice, was evaluated by measuring the increase of the serum creatine kinase $(\mathrm{CK})$.

RESULTS In silico analysis demonstrated the possible profile de interaction of KF498604 and KF498605 clones with CB subnit, and kinetic analysis of interaction determined affinity with $\mathrm{KD}$ value in 81,34 and $1716 \mathrm{nM}$, respectively. Both clones were able to inhibit more than $70 \%$ the in vitro phospholipase activity of the CTX and $\mathrm{CB}$, in a 1:40 ratio (w/w). Additionally, the $\mathrm{KF} 498604$ was capable to neutralize more than $60 \%$ of the myotoxic effects induced by crotoxin-B in mice, in a 1:20 ratio (w/w).

CONCLUSION Preliminary results demonstrate that the selected nanobodies could be an interesting tool to improve the crotalic serum therapy, however in vivo neutralization ability of the neurotoxic effects caused by crotoxin and Crotalus durissus terrificus venom are being investigated.

KEYWORDS VHH, nanobodies, crotoxin-B, PLA2-CB, Crotalus durissus terrificus. 